\title{
On ideals with skew derivations of prime rings
}

\author{
Nadeem ur Rehman and Mohd Arif Raza
}




\title{
ON IDEALS WITH SKEW DERIVATIONS OF PRIME RINGS
}

\author{
NADEEM UR REHMAN AND MOHD ARIF RAZA
}

Received 24 April, 2014

\begin{abstract}
Let $R$ be a prime ring and set $[x, y]_{1}=[x, y]=x y-y x$ for all $x, y \in R$ and inductively $[x, y]_{k}=\left[[x, y]_{k-1}, y\right]$ for $k>1$. We apply the theory of generalized polynomial identities with automorphism and skew derivations to obtain the following result: Let $R$ be a prime ring and $I$ a nonzero ideal of $R$. Suppose that $(\delta, \varphi)$ is a skew derivation of $R$ such that $\delta([x, y])=[x, y]_{n}$ for all $x, y \in I$, then $R$ is commutative.
\end{abstract}

2010 Mathematics Subject Classification: 16N20; 16W25; 16N55; 16N60

Keywords: skew derivation, automorphism, generalized polynomial identity (GPI), prime ring, ideal

\section{Introduction, Notation And Statements of the Results}

Throughout this paper, unless specifically stated, $R$ is always an associative prime ring with center $Z(R), Q$ its Martindale quotient ring. Note that $Q$ is also prime and the center $C$ of $Q$, which is called the extended centroid of $R$, is field (we refer the reader to [1] for the definitions and related properties of these objects). For any $x, y \in R$, the symbol $[x, y]$ stands for the commutator $x y-y x$. Recall that a ring $R$ is called prime if for any $x, y \in R, x R y=\{0\}$ implies that either $x=0$ or $y=0$. An additive mapping $d: R \longrightarrow R$ is called a derivation if $d(x y)=d(x) y+x d(y)$ holds for all $x, y \in R$. An additive mapping $F: R \longrightarrow R$ is called a generalized derivation if there exists a derivation $d: R \longrightarrow R$ such that $F(x y)=F(x) y+x d(y)$ holds for all $x, y \in R$, denoted by $(F, d)$. Hence, the concept of generalized derivations covers both the concepts of a derivation and of a left multiplier.

Given any automorphism $\varphi$ of $R$, an additive mapping $\delta: R \rightarrow R$ satisfying $\delta(x y)=\delta(x) y+\varphi(x) \delta(y)$ for all $x, y \in R$ is called a $\varphi$-derivation of $R$, or a skew derivation of $R$ with respect to $\varphi$, denoted by $(\delta, \varphi)$. It is easy to see if $\varphi=1_{R}$, the identity map of $R$, then a $\varphi$-derivation is merely an ordinary derivation. And if $\varphi \neq 1_{R}$, then $\varphi-1_{R}$ is a skew derivation. Thus the concept of skew derivations can be regard as a generalization of both derivations and automorphism. When $\delta(x)=\varphi(x) b-b x$ for some $b \in Q$, then $(\delta, \varphi)$ is called an inner skew derivation, and otherwise it is outer. Any skew derivation $(\delta, \varphi)$ extends uniquely to a skew derivation of $Q$ [12] via extensions of each map to $Q$. Thus we may assume that any skew derivation of 
$R$ is the restriction of a skew derivation of $Q$. Recall that $\varphi$ is called an inner automorphism if when acting on $Q, \varphi(q)=u q u^{-1}$ for some invertible $u \in Q$. When $\varphi$ is not inner, then it is called an outer automorphism. The skew derivations have been extensively studied by many researchers from various views (see for instance [5] and [12], where further references can be found).

Let $Q_{* C} C\{X\}$ be the free product of $Q$ and the free algebra $C\{X\}$ over $C$ on an infinite set $X$, of indeterminate. Elements of $Q_{* C} C\{X\}$ are called generalized polynomials and a typical element in $Q_{* C} C\{X\}$ is a finite sum of monomials of the form $\alpha a_{i_{0}} x_{j_{1}} a_{i_{1}} x_{j_{2}} \cdots x_{j_{n}} a_{i_{n}}$ where $\alpha \in C, a_{i k} \in Q$ and $x_{j k} \in X$. We say that $R$ satisfies a nontrivial generalized polynomial identity (abbreviated as GPI) if there exists a nonzero polynomial $\phi\left(x_{i}\right) \in Q_{* C} C\{X\}$ such that $\phi\left(r_{i}\right)=0$ for all $r_{i} \in R$. By a generalized polynomial identity with automorphisms and skew derivations, we mean an identity of $R$ expressed as the form $\phi\left(\varphi_{j}\left(x_{i}\right), \delta_{k}\left(x_{i}\right)\right)$, where each $\varphi_{j}$ is an automorphism, each $\delta_{k}$ is a skew derivation of $R$ and $\phi\left(y_{i j}, z_{i k}\right)$ is a generalized polynomial in distinct indeterminates $y_{i j}, z_{i k}$.

We need some well-known facts which will be used in the sequel.

Fact 1 ([5, Theorem 1]). Let $R$ be a prime ring with an automorphism $\varphi$. Suppose that $(\delta, \varphi)$ is a $Q$-outer derivation of $R$. Then any generalized polynomial identity of $R$ in the form $\phi\left(x_{i}, \delta\left(x_{i}\right)\right)=0$ yields the generalized polynomial identity $\phi\left(x_{i}, y_{i}\right)=$ 0 of $R$, where $x_{i}, y_{i}$ are distinct indeterminates.

Fact 2 ([5, Theorem 1]). Let $R$ be a prime ring with an automorphism $\varphi$. Suppose that $(\delta, \varphi)$ is a $Q$-outer derivation of $R$. Then any generalized polynomial identity of $R$ in the form $\phi\left(x_{i}, \varphi\left(x_{i}\right), \delta\left(x_{i}\right)\right)=0$ yields the generalized polynomial identity $\phi\left(x_{i}, y_{i}, z_{i}\right)=0$ of $R$, where $x_{i}, y_{i}, z_{i}$ are distinct indeterminates.

Fact 3 ([14, Proposition]). Let $R$ be a prime algebra over an infinite field $k$ and let $K$ be a field extension over $k$. Then $R$ and $R \otimes_{k} K$ satisfy the same generalized polynomial identities with coefficients in $R$.

The next result is a slight generalization of [13, Lemma 2] and can be obtained directly by the proof of [13, Lemma 2] and Fact 3.

Fact 4 . Let $R$ be a non-commutative simple algebra, finite dimensional over its center $Z$. Then $R \subseteq M_{n}(F)$ with $n>1$ for some field $F$ and $R$ and $M_{n}(F)$ satisfy the same generalized polynomial identities with coefficients in $R$.

In 1992, Daif and Bell [6, Theorem 3], showed that if in a semiprime ring $R$ there exists a nonzero ideal $I$ of $R$ and a derivation $d$ such that $d([x, y])=[x, y]$ for all $x, y \in I$, then $I \subseteq Z(R)$. If $R$ is a prime ring, this implies that $R$ is commutative. Later in 2011, Huang [8, Theorem 2.1], prove that if $R$ is a prime ring, $I$ a nonzero ideal of $R$ and $d$ a derivation of $R$ such that $d([x, y])^{m}=[x, y]_{n}$ for all $x, y \in I$, then $R$ is commutative. At this point the natural question is what happens in case the derivation is replaced by a generalized derivation. In [16], Quadri et. al., generalize 
Daif and Bell result for generalized derivation, they showed that if $R$ is a prime ring, $I$ a nonzero ideal of $R$ and $(F, d)$ a generalized derivation with $d \neq 0$ such that $F([x, y])=[x, y]$ for all $x, y \in I$, then $R$ is commutative. In 2013, Huang and Davvaz [9], generalized Quadri et. al., results, more precisely they proved that if $R$ be a prime ring, $m, n$ are fixed positive integers, and $(F, d)$ a generalized derivation with $d \neq 0$ such that $(F([x, y]))^{m}=[x, y]^{n}$ for all $x, y \in R$, then $R$ is commutative.

Here we will continue the study of analogue problems on ideals of a prime ring involving skew derivations. The goal of this paper is to extend Daif and Bell theorem [6], and Huang theorem [8], in a systematic way by using the theory of generalized polynomial identities with automorphisms and skew derivations as developed by Kharchenko [11], Chuang [3,4] and recently by Chuang and Lee [5].

Explicitly we shall prove the following theorem.

Theorem 1. Let $R$ be a prime ring, $I$ a nonzero ideal of $R$ and $n$ a fixed positive integer. Suppose that $(\delta, \varphi)$ is a skew derivation of $R$ such that $\delta([x, y])=[x, y]_{n}$ for all $x, y \in I$, then $R$ is commutative.

When $\delta=\varphi-1_{R}$, we obtain the following

Corollary 1. Let $R$ be a prime ring, I a nonzero ideal of $R$, and $n$ a fixed positive integer. If $\varphi$ is a non-identity automorphism of $R$ such that $\varphi([x, y])=[x, y]_{n}$ for all $x, y \in I$, then $R$ is commutative.

Let $R$ be a unital ring. For a unit $u \in R$, the map $\varphi_{u}: x \rightarrow u x u^{-1}$ defines an automorphism of $R$. If $d$ is a derivation of $R$, then it is easy to see that the map $u d: x \rightarrow u d(x)$ defines a $\varphi_{u}$-derivation of $R$. So we have

Corollary 2. Let $R$ be a prime unital ring, $u$ a unit in $R, I$ a nonzero ideal of $R$, and $n$ a fixed positive integer. Suppose that $\varphi_{u}$ is a derivation of $R$ such that $\varphi_{u}([x, y])=[x, y]_{n}$ for all $x, y \in I$, then $R$ is commutative.

\section{MAin RESUlt}

Now, we are in a position to prove the main result:

Theorem 2. Let $R$ be a prime ring, I a nonzero ideal of $R$ and $n$ a fixed positive integer. Suppose that $(\delta, \varphi)$ is a skew derivation of $R$ such that $\delta([x, y])=[x, y]_{n}$ for all $x, y \in I$, then $R$ is commutative.

Proof. If $\delta=0$, then $[x, y]_{n}=0$ for all $x, y \in I$, which can be rewritten as

$$
[x, y]_{n}=0=\left[I_{x}(y), y\right]_{n-1} \text { for all } x, y \in I .
$$

By Lanski [13, Theorem 1], either $R$ is commutative or $I_{x}=0$, i.e., $I \subseteq Z(R)$ in which case $R$ is also commutative by Mayne [15, Lemma 3].

Now we assume that $\delta \neq 0$ and $\delta([x, y])=[x, y]_{n}$ for all $x, y \in I$, which can be rewritten as

$$
(\delta(x) y+\varphi(x) \delta(y))-(\delta(y) x+\varphi(y) \delta(x))=[x, y]_{n} .
$$


In the light of Kharchenko's theory [11], we split the proof into two cases:

Case 1. Let $\delta$ is $Q$-outer, then $I$ satisfies the polynomial identities

$$
(s y+\varphi(x) t)-(t x+\varphi(y) s)=[x, y]_{n}, \text { for all } x, y, s, t \in I .
$$

Firstly, we assume that $\varphi$ is not $Q$-inner, then for all $x, y, s, t, u, v \in I$, we have

$$
(s y+u t)-(t x+v s)=[x, y]_{n} \text {, for all } x, y, s, t, u, v \in I .
$$

In particular $s=t=0$, then $I$ satisfied the polynomial identity $[x, y]_{n}=0$, for all $x, y \in I$, so by Lanski [13, Theorem 1], $R$ is commutative.

Secondly, if $\varphi$ is $Q$-inner, then there exist an invertible element $T \in Q, \varphi(x)=$ $T x T^{-1}$ for all $x \in R$. Thus from (2.2), we have

$$
\left(s y+T x T^{-1} t\right)-\left(t x+T y T^{-1} s\right)=[x, y]_{n} \text { for all } x, y, s, t \in I .
$$

In particular $s=t=0$, and using the same argument presented as above, $R$ is commutative.

Case 2. Let $\delta$ is $Q$-inner, then $\delta(x)=\varphi(x) q-q x$ for all $x \in R, q \in Q$. From (2.1), we have

$$
\begin{gathered}
(\varphi(x) q-q x) y+\varphi(x)(\varphi(y) q-q y)-(\varphi(y) q-q y) x-\varphi(y)(\varphi(x) q-q x) \\
=[x, y]_{n} \text { for all } x, y \in I .
\end{gathered}
$$

If $\varphi$ is not $Q$-inner, then $I$ satisfies the polynomial identity

$$
\begin{aligned}
(u q-q x) y+u(v q-q y) & -(v q-q y) x-v(u q-q x) \\
= & {[x, y]_{n} \quad \text { for all } x, y, u, v \in I . }
\end{aligned}
$$

In particular $u=v=0$, then $I$ satisfied the following polynomial identity

$$
(-q x y+q y x)=[x, y]_{n} \text {, for all } x, y \in I \text {. }
$$

By Chuang [5, Theorem 1 and Theorem 2], shows that $Q$ satisfies this polynomial identity and hence $R$ as well. Note that this is a polynomial identity and hence there exist a field $\mathbb{F}$ such that $R \subseteq M_{k}(\mathbb{F})$, the ring of $k \times k$ matrices over a field $\mathbb{F}$, where $k \geq 1$. Moreover, $R$ and $M_{k}(\mathbb{F})$ satisfy the same polynomial identity[2], that is $M_{k}(\mathbb{F})$ satisfy

$$
(q y x-q x y)=[x, y]_{n} .
$$

Denote $e_{i j}$ the usual matrix unit with 1 in $(i, j)$-entry and zero elsewhere. By choosing $x=e_{12}, y=e_{22}, q=e_{12}$, we see that

$$
\begin{aligned}
0=(q[y, x])-[x, y]_{n} & =\left(e_{12}\left[e_{22}, e_{12}\right]\right)-\left[e_{12}, e_{22}\right]_{n} \\
& =-e_{12} \neq 0, \text { a contradiction. }
\end{aligned}
$$

Now consider, if $\varphi$ is $Q$-inner, then there exist an invertible element $T \in Q, \varphi(x)=$ $T x T^{-1}$ for all $x \in R$. From (2.3) we can write,

$$
\begin{aligned}
\left(T x T^{-1} q-q x\right) y & +T x T^{-1}\left(T y T^{-1} q-q y\right)-\left(T y T^{-1} q-q y\right) x \\
& -T y T^{-1}\left(T x T^{-1} q-q x\right)=[x, y]_{n} \text { for all } x, y \in I .
\end{aligned}
$$


We can see easily that if $T^{-1} q \in C$, then

$\delta(x)=T x T^{-1} q-q x=T\left(x T^{-1} q-T^{-1} q x\right)=T\left[x, T^{-1} q\right]=0$, a contradiction.

Thus $T^{-1} q \notin C$. with this,

$$
\begin{aligned}
\phi(x, y)= & \left(T x T^{-1} q-q x\right) y+T x T^{-1}\left(T y T^{-1} q-q y\right) \\
& -\left(T y T^{-1} q-q y\right) x-T y T^{-1}\left(T x T^{-1} q-q x\right)-[x, y]_{n} .
\end{aligned}
$$

Since by [2] or [1, Theorem 6.4.4], $I$ and $Q$ satisfy the same generalized polynomial identities, with this we can see easily that $\phi(x, y)=0$ is a nontrivial generalized polynomial identity of $Q$. Let $\mathscr{F}$ be the algebraic closure of $C$ if $C$ is infinite, otherwise let $\mathcal{F}$ be $C$. By Fact $3, \phi(x, y)$ is also a generalized polynomial identity of $Q \otimes_{C} \mathcal{F}$. Moreover, in view of [7, Theorem 3.5], $Q \otimes_{C} \mathcal{F}$ is a prime ring with $\mathscr{F}$ as its extended centroid. Thus $Q \otimes_{C} \mathscr{F}$ is a prime ring satisfies a nontrivial generalized polynomial identity and its extended centroid $\mathscr{F}$ is either an algebraically closed field or a finite field. Since both $Q$ and $Q \otimes_{C} \mathcal{F}$ are prime and centrally closed [7, Theorem 3.5], we may replace $R$ by $Q$ or $Q \otimes_{C} \mathcal{F}$. Thus we may assume that $R$ is centrally closed and the field $\mathscr{F}$ which is either algebraically closed or finite and $R$ satisfies generalized polynomial identity (2.4). By Martindale's theorem [1, Corollary 6.1.7], $R$ is a primitive ring having nonzero socle with the field $\mathscr{D}$ as its associated division ring. By Jacobson theorem [10, p.75], $R$ is isomorphic to a dense subring of the ring of linear transformations on a vector space $V$ over $\mathscr{D}$ (or $\operatorname{End}\left(V_{\mathscr{D}}\right)$ in brief), containing nonzero linear transformations of finite rank.

We assume that $\operatorname{dim}\left(V_{\mathscr{D}}\right) \geq 2$, otherwise we are done.

Step 1. We want to show that $w$ and $T^{-1} q w$ are linearly $\mathcal{D}$-dependent for all $w \in \mathcal{V}$. If $T^{-1} q w=0$ then $\left\{w, T^{-1} q w\right\}$ is linearly $\mathcal{D}$-dependent. Suppose on contrary that $w_{0}$ and $T^{-1} q w_{0}$ are linearly $\mathscr{D}$-independent for some $w_{0} \in \mathscr{D}$.

If $T^{-1} w_{0} \notin \operatorname{Span}_{\mathscr{D}}\left\{w_{0}, T^{-1} q w_{0}\right\}$ then $\left\{w_{0}, T^{-1} q w_{0}, T^{-1} w_{0}\right\}$ are linearly $\mathscr{D}$ independent. By the density of $R$ there exist $x, y \in R$ such that

$$
\begin{aligned}
& x w_{0}=0, \quad x T^{-1} q w_{0}=T^{-1} w_{0}, \quad x T^{-1} w_{0}=0 \\
& y w_{0}=w_{0}, \quad y T^{-1} q w_{0}=0, \quad y T^{-1} w_{0}=T^{-1} w_{0} .
\end{aligned}
$$

With all these, we obtained from (2.4),

$$
\begin{aligned}
-w_{0}= & \left(\left(T x T^{-1} q-q x\right) y+T x T^{-1}\left(T y T^{-1} q-q y\right)-\left(T y T^{-1} q-q y\right) x\right. \\
& \left.-T y T^{-1}\left(T x T^{-1} q-q x\right)-[x, y]_{n}\right) w_{0}, \text { a contradiction. }
\end{aligned}
$$

If $T^{-1} w_{0} \in \operatorname{Span}_{\mathscr{D}}\left\{w_{0}, T^{-1} q w_{0}\right\}$ then $T^{-1} w_{0}=w_{0} \beta+T^{-1} q w_{0} \gamma$ for some $\beta, \gamma \in \mathscr{D}$ and $\beta \neq 0$. Since $w_{0}$ and $T^{-1} q w_{0}$ are linearly $\mathscr{D}$-independent, by the density of $R$ there exist $x, y \in R$ such that

$$
\begin{array}{ll}
x w_{0}=0, & x T^{-1} q w_{0}=w_{0} \beta+T^{-1} q w_{0} \gamma \\
y w_{0}=w_{0}, & y T^{-1} q w_{0}=0 .
\end{array}
$$


The application of (2.4) implies that

$$
\begin{aligned}
0= & \left(\left(T x T^{-1} q-q x\right) y+T x T^{-1}\left(T y T^{-1} q-q y\right)-\left(T y T^{-1} q-q y\right) x\right. \\
& \left.-T y T^{-1}\left(T x T^{-1} q-q x\right)-[x, y]_{n}\right) w_{0}=-T w_{0} \beta=-w_{0} \beta \neq 0,
\end{aligned}
$$

and we arrive at a contradiction. So we conclude that $\left\{w_{0}, T^{-1} w_{0}\right\}$ are linearly $\mathscr{D}$ dependent, for all $w_{0} \in \mathcal{V}$ as claimed.

Step 2. By using the arguments presented above, we prove that $T^{-1} q w_{0}=w_{0} \mu(w)$, for all $w \in \mathcal{V}$, where $\mu(w) \in \mathscr{D}$ depends on $w \in \mathcal{V}$. In fact, it is easy to check that $\mu(w)$ is independent of choice $w \in \mathcal{V}$. Indeed, for any $w, z \in \mathcal{V}$, in view of above situation, there exist $\mu(w), \mu(z), \mu(w+z) \in \mathscr{D}$ such that

$$
T^{-1} q w=w \mu(w), T^{-1} q z=z \mu(z), T^{-1} q(w+z)=(w+z) \mu(w+z)
$$

and therefore,

$$
w \mu(w)+z \mu(z)=T^{-1} q(w+z)=(w+z) \mu(w+z) .
$$

Hence,

$$
w(\mu(w)-\mu(w+z))+z(\mu(z)-\mu(w+z))=0 .
$$

Since $w$ and $z$ are $\mathscr{D}$-independent, then $\mu(w)=\mu(z)=\mu(w+z)$. Otherwise, $w$ and $z$ are $\mathscr{D}$-dependent, say $w=\lambda z$ for some $\lambda \in \mathscr{D}$. Thus,

$$
w \mu(w)=T^{-1} q w=T^{-1} q \lambda z=\lambda T^{-1} q z=\lambda z \mu(z)=w \mu(z)
$$

i.e., $\mathcal{V}(\mu(w)-\mu(z))=0$. Since $\mathcal{V}$ is faithful, we get $\mu(w)=\mu(z)$. Hence, we conclude that there exists $\chi \in \mathscr{D}$ such that $T^{-1} q w=w \chi$ for all $w \in \mathcal{V}$.

At last, we want to show that $\chi \in Z(\mathscr{D})$ (the center of $\mathscr{D}$ ). Indeed, for any $\eta \in \mathscr{D}$, we have

and on the other hand,

$$
T^{-1} q(w \eta)=(w \eta) \chi=w(\eta \chi)
$$

$$
T^{-1} q(w \eta)=\left(T^{-1} q w\right) \eta=(w \chi) \eta=w(\chi \eta) .
$$

Therefore, $\mathcal{V}(\eta \chi-\chi \eta)=0$ and thus, $\eta \chi=\chi \eta$, which implies that $\chi \in Z(\mathscr{D})$. Hence, $T^{-1} q \in C$, a contradiction. With this completes the proof of the theorem.

The following example demonstrates that the hypothesis of primeness of $R$ is essential in Theorem 1.

Example 1. Let $S$ be the set of all integers. Consider

$R=\left\{\left(\begin{array}{cc}a & b \\ 0 & 0\end{array}\right) \mid a, b \in S\right\}$ and $I=\left\{\left(\begin{array}{cc}0 & b \\ 0 & 0\end{array}\right) \mid b \in S\right\}$. Define maps $\varphi: R \rightarrow$ $R$ by $\varphi\left(\begin{array}{ll}a & b \\ 0 & 0\end{array}\right)=\left(\begin{array}{cc}a & -b \\ 0 & 0\end{array}\right)$ and $\delta: R \rightarrow R$ by $\delta\left(\begin{array}{cc}a & b \\ 0 & 0\end{array}\right)=\left(\begin{array}{cc}a & -2 b \\ 0 & 0\end{array}\right)$. The fact that $\left(\begin{array}{ll}0 & 1 \\ 0 & 0\end{array}\right) \neq 0$ and $\left(\begin{array}{ll}0 & 1 \\ 0 & 0\end{array}\right) R\left(\begin{array}{ll}0 & 1 \\ 0 & 0\end{array}\right)=0$ implies that $R$ is not 
prime. It is easy to check that $I$ is a nonzero ideal of $R$ and $(\delta, \varphi)$ is a skew derivation of $R$ such that $\delta([x, y])=[x, y]_{n}$ for all $x, y \in I$. However, $R$ is not commutative.

Remark 1. In view of the above result, it is an obvious question, what about the commutativity of $R$, if $\delta([x, y])^{m}=[x, y]_{n}$ for all $x, y \in I$ (or a Lie ideal $L$ ). Unfortunately, we are unable to solve it and leave as an open question whether or not this result can be prove.

\section{REFERENCES}

[1] K. I. Beidar, W. S. Martindale III, and A. V. Mikhalev, Rings with Generalized Identities. New York: Pure and Applied Mathematics, Marcel Dekker 196, 1996.

[2] C. L. Chuang, "GPI's having coefficients in utumi quotient rings," Proc. Amer. Math. Soc., vol. 103, pp. 723-728, 1988.

[3] C. L. Chuang, "Differential identities with automorphism and anti-automorphism-i," J. Algebra, vol. 149, pp. 371-404, 1992.

[4] C. L. Chuang, "Differential identities with automorphism and anti-automorphism-ii," J. Algebra, vol. 160, pp. 291-335, 1993.

[5] C. L. Chuang and T. K. Lee, "Identities with a single skew derivation," J. Algebra, vol. 288, pp. 59-77, 2005.

[6] M. N. Daif and H. E. Bell, "Remarks on derivations on semiprime rings," Internt. J. Math. and Math. Sci., vol. 15, pp. 205-206, 1992.

[7] T. S. Erickson, W. S. Martindale 3rd, and J. M. Osborn, "Prime nonassociative algebras," Pacific. J. Math., vol. 60, pp. 49-63, 1975.

[8] S. Huang, "Derivation with engel conditions in prime and semiprime rings," Czechoslovak Math. J., vol. 61, no. 136, pp. 1135-1140, 2011.

[9] S. Huang and B. Davvaz, "Generalized derivations of rings and banach algebras," Communication in algebra, vol. 41, pp. 1188-1194, 2013.

[10] N. Jacobson, Structure of rings. Rhode Island: Amer. Math. Soc. Colloq. Pub. 37, 1964.

[11] V. K. Kharchenko, "Generalized identities with automorphisms," Algebra Logic, vol. 14, no. 2, pp. 132-148, 1975.

[12] V. K. Kharchenko and A. Z. Popov, "Skew derivations of prime rings," Comm. Algebra, vol. 20, pp. 3321-3345, 1992.

[13] C. Lansk, "An engel condition with derivation," Proc. Amer. Math. Soc., vol. 118, pp. 75-80, 1993.

[14] P. H. Lee and T. L. Wong, "Derivations cocentralizing lie ideals," Bull. Inst. Math. Acad. Sin., vol. 23, pp. 1-5, 1995.

[15] J. H. Mayne, "Centralizing mappings of prime rings," Can. Math. Bull., vol. 27, pp. 122-126, 1984.

[16] M. A. Quadri, M. S. Khan, and N. Rehman, "Generalized derivations and commutativity of prime rings," Indian J. Pure Appl. Math., vol. 34, no. 98, pp. 1393-1396, 2003.

Authors' addresses

Nadeem ur Rehman

Department of Mathematics, Aligarh Muslim University, 202002, Aligarh, India

E-mail address: nu.rehman.mmeamu.ac.in 
Mohd Arif Raza

Department of Mathematics, Aligarh Muslim University, 202002, Aligarh, India

E-mail address: arifraza03@gmail.com 